\title{
Urinary tract infections in obese children and adolescents with their outcomes and clinical aspects
}

\author{
Semra Şen, MD ${ }^{1}$, Deniz Özalp Kızılay, MD ${ }^{2}$, Betül Ersoy, MD ${ }^{2}$ \\ 1 Department of Pediatric Infectious Diseases, Celal Bayar University Hospital, Manisa, Turkey \\ 2 Department of Pediatric Endocrinology, Celal Bayar University Hospital, Manisa, Turkey.
}

*Corresponding Author: Dr. Semra Şen, MD, Division of Pediatric Infections, Department of Pediatrics, Medical University of Celal Bayar, Manisa Tel.: 905057278411, Email: drsemrasen@gmail.com

\begin{abstract}
Aim: The relationship between obesity and urinary tract infections (UTI) was reported in adult studies, but pediatric data is lacking. Methods: This prospective study included children, who were admitted to the outpatient clinic only with the complaint of obesity. UTI screening with symptomatic questioning and clinical feature was performed. Symptomatic participants were examined(urinalysis, culture, or/and blood work, imaging tests). Characteristics of those with UTI, and we determined an obese/overweight, but non-UTI control group matched with age and gender and conducted a case-control study. Results: In this study, 1001 participants were screened, UTI frequency was 3,39\%, with $91,2 \%$ female predominance. The mean age of all participants was $11.44 \pm 3.21$ years. All of the UTIs were lower tract infections. Escherichia coli(82,4\%) was the most frequently isolated microorganism. History of UTI and hypertension was more frequent in the UTI group( $n=34)$ compared with non UTI group $(n=136)(p=0,001 ; p=0,001)$. Conclusion: In the UTI group, hypertension and previous UTI history were shown to be more common, so it may be important to question obese/overweight children for UTI at their clinical application.
\end{abstract}

Keywords: Obesity, Child, Urinary tract infections.

\section{INTRODUCTION}

The increasing tendency to obesity in children and adolescents is considered a severe public health problem associated with complications such as diabetes mellitus and cardiovascular diseases ${ }^{[1]}$. The relationship between obesity and many types of infections was also reported in adult studies. However, data for children and adolescents is lacking. In adult studies, it was reported that some confounders, that increase the risk of infection, such as concomitant long-term diabetes mellitus, may affect the direct relationship of obesity and infections. The frequency of such confounders is lower in children, therefore pediatric studies are more considerable to reveal the relationship between infections and obesity ${ }^{[1-2]}$.

Several studies, generally based on hospital records, mostly in children under the age of five, have suggested that pediatric obesity could be associated with the development of urinary tract infection (UTI) ${ }^{[3-8]}$. However, little is known about the frequency of UTI in obese children and adolescents and the potential effects of obesity on the clinical outcomes of UTI. This study aims to evaluate the frequency and clinical aspects of UTI in obese/overweight children and adolescents.

\section{METODS}

\section{Study design}

\section{Study population}

This prospective study was conducted in Manisa Celal Bayar UniversityHospital, between January 1, 2016, and December 31, 2017. We performed UTI screening with a questionnaire that inquired about UTI symptoms and clinical features in all obese / overweight children and adolescents who admitted to the General Pediatric and Pediatric Endocrinology outpatient clinic. The detailed content of the questionnaire applied to the patients were age, gender, urinary tract infection symptoms (dysuria, frequency, urgency, incontinence, hematuria, suprapubic pain, fever, abdominal pain), urinary tract infection risk factors (urinary retention behavior, voiding dysfunction [in filling and / or emptying the bladder] and the history of parasite, antibiotic use, diabetes mellitus, catheterization and constipation), and the history of Previous and Recurrent UTI.Routine urinalysis was requested from patients with 
suspected urinary tract infection in the questionnaire responses.Urine culture was requested from those who had evidence of infection in urinalysis. This stage of the study was a cross-sectional and frequency determination study. The questionnaire was applied to a total of 1001 obese and overweight children, and 34 patients were diagnosed with urinary culture-proven UTI. In addition, in order to compare the clinical and laboratory characteristics of patients with and without UTI,34 patients who were confirmed UTI by urine culture were defined as case group and 136 obese and overweight children without UTI were randomly selected as the control group.

Children with type 1 diabetes or obesity with a syndrome (Prader-Willi syndrome, Laurence Moon Biedl syndrome, etc.) or endocrinologic pathologies such as Cushing's syndrome were excluded. Children with kidney or other systemic diseases and contaminated urine cultures (defined as mixed growth bacteria ${ }^{[9]}$ ) were also excluded from the study. The children and their families were informed about the study and written informed consent was obtained. The local ethics committee approved the study.

\section{Clinical and laboratory evaluation}

All overweight/obese children underwent a thorough physical examination and laboratory evaluation. The clinical assessments were all performed by a specially trained clinical research staff.Anthropometric measurements [height and body weight] were performed by using the same toolswhile participants were wearing a light robe and no shoes. Findings for pubertal development were evaluated according to Tanner's staging (Tanner, J.M., Whitehouse, R.H. (1976). Clinical longitudinal standards for height, weight, height velocity, weight velocity, and stages of puberty. Arch. Dis. Child. 51, 170-179.). A testicular volume of $\geq 4 \mathrm{~mL}$ in males, and breast development of stage 2 and over in females, were considered to be findings of puberty. Blood pressure was taken in the supine position, after a ten-minute rest, using the right arm, with a calibrated sphygmomanometer and the appropriate cuff. Systolic blood pressure (SBP), and diastolic blood pressure (DBP) were measured twice and the mean of these two $\mathrm{BP}$ values were taken into consideration.

Blood samples were taken after 10-12 h of night fasting for glucose and insülin. Hemoglobin $\mathrm{A} 1 \mathrm{c}(\mathrm{HbA1c})$ measurement and oral glucose tolerance test (OGTT) were performed in patients with a fasting blood glucose (FPG)>126 mg / dL. During OGTT,blood glucose and insulin concentrations were measured before and at $30,60,90$, and 120 minutes after the consumption of a glucose load in a dose of $1.75 \mathrm{~g}$ per kilogram of body weight (up to a maximum of $75 \mathrm{~g}$ of glucose).

Urine samples for routine urinalysis and urine microscopy were collected from all patients with UTI symptoms. Supportive findings for UTI in urinalysis or urine microscopy were positive nitrite, positive leukocyte esterase, pyuria ( $>5 \mathrm{WBC} / \mathrm{mm} 3)$, hematuria ( $>5$ erythrocytes $/ \mathrm{mm} 3$ ) and bacteriuria. Clean catch mid-stream void urine culture was obtained from symptomatic patients with findings supporting UTI in urinalysis or urine microscopy. The presence of more than $10^{5} \mathrm{CFU} / \mathrm{ml}$ bacteria in clean voided urineculture was considered a positive result for urinary tract infection.

\section{Classification}

We calculated the BMI as weight $(\mathrm{kg})$ divided by square of height $\left(\mathrm{m}^{2}\right)$. BMI-SDS and BMI percentiles were calculated using age and gender-specific norms published by the Center for Disease Control and Prevention (CDC-2000). Obesity was defined as $\mathrm{BMI} \geq 95$ th percentile, and overweight was defined as $\mathrm{BMI} \geq 85$ th for age and sex ${ }^{[11]}$.

Hypertension was defined as a value above the 95th percentile for age and height, according to the National Health and Nutrition Examination Survey ${ }^{[12]}$.

Insulin resistance (IR) was evaluated according to the homeostasis model assessment insulin resistance (HOMA-IR) index. Cut-off values for different stages were prepubertal $>2.5$, pubertal>4 [11]. Type 2 diabetes was diagnosed in patients whose $\mathrm{HbA} 1 \mathrm{c}$ was measured $>6.5 \%$ and whose plasma glucose level was $>200 \mathrm{mg} / \mathrm{dL}$ at the 2 nd hour after OGTT [10].

UTI was defined as significant bacteriuria of a clinically relevant uropathogen in a symptomatic patient.Upper (infection of the kidney) and lower(infection of the kidney bladder, urethra) UTI classifications were performed in patients with UTI.Recurrent UTI was defined as three or more cystitis or two pyelonephritis or one pyelonephritis and one cystitis [9]. This definition was made by examining the hospital records and/or the previous examination results (if available) of patients diagnosed with UTI.

\section{Statistical analysis}

Between-group comparison for categorical variables was performed by using the $\mathrm{x} 2$ test, or Fisher's exact tests.All data were tested for normality using the Kolmogorov-Smirnov test. Mann-Whitney $U$ test was used for comparison of not normally distrubuted continuous and nonparametric variables, T-test was used for normally distrubuted variables. Multivariable logistic regression analyses were performed to test independent predictors of UTI in obese children and adolescents.

Statistical analyses were performed using Statistical Package for Social Sciences 15.0 (SPSS 15.0) program.P values $<0.05$ were considered statistically significant.

\section{RESULTS}

\section{Characteristics of all obese/overweight participants}

In this study, 1001 obese and overweight children and adolescents were screened (34 (3.39\%) with UTI; 967 (96.6\%) without UTI). The mean age of the all participants $(n=1001)$ was $11.44 \pm 3.21$ years (Median: 12, min-max: 5-18); 594 (59.4\%) were female and 407 (40.7\%) were male. The median age of UTI group was significantly younger than non-UTI participants [10 years $(\min -\max : 5-16)$ vs. 12.5 years $(\min -\max : 5-18)](P=$ 
0.000). Patients with UTI were $91.2 \%$ female and $8.8 \%$, male. UTI was significantly more common in females ( $n=31$ UTI/594) than males $(n=3 U T I / 407)(P=0.002)$. There was no significant difference between the two groups in terms of BMI values [median BMI in UTI group 25.4 (19.11-41); median BMI in nonUTIs was 27.7 (18.9-51)] $(P=0.074)$. Complete urine analysis of the patients revealed pyuria $(n=19)$, microscopic hematuria $(n=5)$, bacteriuria $(n=5)$ and nitrite positivity $(n=5)$. The most frequently isolated agent in urine culture was Escherichia coli $(82.4 \%)$. All of the patients with UTI were lower tract UTI and were treated orally. All patients with UTI underwent renal USG, hydronephrosis was detected in four patients and nephrolithiasis in one patient.

\section{Characteristics of obese/overweight participants with UTI and control group}

Risk factors and urinalysis variables were compared in obese/overweight children with UTI $(n=34)$, and control group $(n=136)$. There was no statistical difference between the two groups in terms of age and gender. The results are presented in Table 1. Twenty four of the patients with UTI $(71.6 \%)$ had previous UTIs. Ten (29.4\%)patients had first UTI, and 24 (71.6\%) patients had recurrent UTI. Previous UTI history was significantly higher in the UTI group $(73.52 \%)$ compared to the control group (42.64\%) $(\mathrm{p}=0.001)$. Recurrent UTI was significantly higher in UTI group (50\%) compared to the control group (25\%) $(P=0.047)$. Hypertension was significantly higher in the UTI group compared to the control group $(P=0.001)$.

None of the participants had a history of catheterization or antibiotics use. Only two participants in control group had a diagnosis of type 2 diabetes. Other risk factors such as urineholding behavior, voiding dysfunction (abnormalities in either the filling and/or emptying of the bladder), parasite, antibiotic use, diabetes mellitus, for UTI are presented in Table 1. UTIs were not associated with fasting glucose, fasting insulin, HOMA-IR (Table 1). The multivariable logistic regression model included recurrent UTI, previous UTI, and hypertension. In the logistic regression model, there was no independent predictor for developing UTI in obese/overweight patients $(P=0.710)$.

We compared additionally hypertensive participants $(n=6)$ with normotensive obese/overweight participants $(n=164)$. Participants in the UTI group were higher in the hypertension group than the normotensive group $(P=0.000)$. Although not statistically significant previous and recurrent UTI history was higher in hypertensive than those who were normotensive (83.3\% vs. $47.7 \%, P=0.111)$, ( $50 \%$ vs. $30.48 \%, P=0.377$ ). Also, DM was higher in the hypertensive group (16.6\% vs. $2.43 \%)$ $(P=0.167)$. Logistic regression analysis was not performed because the number of hypertensive patients was less than 10 .

All microorganisms identified in urine culture are presented in Table 2.

Table 1; Demographic data and UTI risk factors, clinical and laboratory characteristics of the patients

\begin{tabular}{|c|c|c|c|}
\hline Subjects (n) & UTI group $(n=34)$ & Control group $(n=136)$ & $\mathbf{P}$ \\
\hline Age (years) median (minimum-maximum) & $10(5-16)$ & $10(5-16)$ & 0.635 \\
\hline Mean $\pm S D$ & $10.27 \pm 2.96$ & $10.57 \pm 2.71$ & \\
\hline Gender Female & $31(91.17 \%)$ & $124(91.17 \%)$ & 1 \\
\hline Obesity/Overweight & $31 / 3$ & $124 / 12$ & 0.739 \\
\hline Prepubertal/pubertal & $11 / 23$ & $40 / 96$ & 0.738 \\
\hline Weight (kg) & $54(29-105)$ & $56.3(25.5-128)$ & 0.782 \\
\hline Height $(\mathrm{cm})$ & $145(114-167)$ & $146(110.5-174.8)$ & 0.410 \\
\hline BMI $\left(\mathrm{kg} / \mathrm{m}^{2}\right)$ & $25.4(19.11-41)$ & $25.9(18.9-47.6)$ & 0.761 \\
\hline BMI persantil (\%) & 98.44 (89.43-99.9) & 98.07 (85.86-99.9) & 0.220 \\
\hline BMI SDS $\left(\mathrm{kg} / \mathrm{m}^{2}\right)$ & $2.12(1.25-2.83)$ & $2.06(1.07-2.86)$ & 0.310 \\
\hline Previous UTI (n, \%) & $25(73.52 \%)$ & $58(42.64 \%)$ & 0.001 \\
\hline Recurrent UTI (n, \%) & $17(50 \%)$ & $34(25 \%)$ & 0.047 \\
\hline Diabetes mellitus ( $\mathrm{n}, \%)$ & $0(0 \%)$ & $2(1.4 \%)$ & 0.490 \\
\hline Hypertension (n, \%) & $5(14.7 \%)$ & $1(0.73 \%)$ & 0.001 \\
\hline Insulin resistance(n, \%) & $17(50 \%)$ & $56(41.17 \%)$ & 0.901 \\
\hline Constipation (n, \%) & $11(32.35 \%)$ & $30(22.05 \%)$ & 0.363 \\
\hline Voiding dysfunction (n, \%) & $7(20.58 \%)$ & $12(8.82 \%)$ & 0.134 \\
\hline Urine-holding behavior(n, \%) & $17(50 \%)$ & $48(35.29 \%)$ & 0.265 \\
\hline Parasite $(n, \%)$ & $1(2.94 \%)$ & $13(9.55 \%)$ & 0.306 \\
\hline Hygiene problems (n, \%) & $1(2.94 \%)$ & $10(7.35 \%)$ & 0.458 \\
\hline Fasting glucose (mg/dL) & $84(70-98)$ & $86(52-107)$ & 0,429 \\
\hline Fasting insulin (mUI/L) & $19,73(5,52-36,2)$ & $23,3(3,3-64,63)$ & 0,299 \\
\hline HOMA-IR & $4,08(1,29-8,30)$ & $4,56(1,92-13,87)$ & 0,269 \\
\hline
\end{tabular}

Abbreviations: BMI, body mass index; HOMA-IR, homeostatic model assessment of insulin resistance; UTI, urinary tract infection. 
Table 2: Microorganisms Isolated from Urinary Cultures

\begin{tabular}{|l|l|l|}
\hline Organism & No. & Percent (\%) \\
\hline Escherichia coli & 28 & 82,4 \\
\hline Klebsiella pneumoniae & 5 & 14,7 \\
\hline Citrobacter farmeri & 1 & 2,9 \\
\hline & 34 & $100 \%$ \\
\hline
\end{tabular}

\section{DISCUSSION}

In this study, all obese/overweight children who applied to the endocrinology outpatient clinic for examination due to obesity were questioned for UTI symptoms. In this study, the frequency of UTI in obese/overweight children and adolescents was $3.39 \%$. All cases were lower UTI which was more common in girls. In UTI cases, the previous history of UTI and presence of hypertension were significantly higher than those without UTI. Hypertension in the UTI group may be associated with recurrent UTIs in the patient group.This study reveals the importance of questioning obese patients in terms of both recurrent UTI and UTI symptoms.

There are differences in the literature regarding the prevalence and incidence of UTI in pediatric age groups. This may be due to presentations ranging from the absence of specific urinary symptoms to fulminant urosepsis in different age groups. A pediatric metanalysis reported that the prevalence of UTI varies by age and race ${ }^{[13]}$. However, Urologic Disease in America project study revealed that UTIs affect $2.4 \%-2.8 \%$ of all children's age groups yearly ${ }^{[14]}$. UTIs were reported in $1 \%$ of prepubertal boys and $3 \%$ of prepubertal girls, according to the European Association of Urology ${ }^{[15]}$. In a review, the annual incidence of a UTI between $6-16$ years was reported $0.7 \%$ to $2.3 \%$ for girls and $0.04 \%$ to $0.2 \%$ for boys ${ }^{[16]}$. There are few studies on obesity and UTI in literature. In a study, according to the data obtained from the 41819 obese patient records, 1425 (2.9\%) (1248 female / 177 male) obese children aged between 2-20 years were diagnosed as UTI. Obese females were $45 \%$ more likely to have a UTI compared with their non-obese female control group, while obese males had a $10 \%$ increased risk [8]. Although studies in the literature focus on UTI in obese patients under two years of age, there are very few studies on children over 2 years old. Also, publications investigating the relationship between UTI and obesity are often based on retrospective records of hospital admission diagnoses ${ }^{[4-7]}$. In our study, based on the follow-up of obese patients, the frequency of UTI was found to be $3.39 \%$ in the obese population aged $5-18$ years. Patients diagnosed with UTI were mostly in the pubertal period(67.64\%). In our study population who have applied only with complaints of obesity and were questioned for UTI symptoms, lower UTI is an expected result. In other studies in the literature, obesity was generally evaluated in patients presenting for lower and upper UTI. Therefore, a considerable rate was determined in our study.

Obesity and UTI association may be due to pelvic floor damage.
In a study investigating the effects of obesity on the pelvic floor, chronic increase in intra-abdominal pressure, pelvic muscle system damage, and nerve conduction disorders were presented as the cause of UTI in adults ${ }^{[25]}$.

Some studies are trying to explain the relationship between obesity and infection. Obesity was reported with reduced activity of the host immune system associated with increased risk of infections. Especially studies on leptin deficiency have shown increased susceptibility to infections ${ }^{[1,17]}$. Considerable failure in phagocytic and oxidative burst activity of monocytes, increased thymic aging, decreased $T$ cell repertoire diversity were reported ${ }^{[18-19]}$. TNF- $\alpha$ was shown to be higher in the obese compared with the nonobese people. Systemic effects of leptin or other adipokines on macrophages and lymphocytes may be the cause risponsible. TNF-a has two clinically important effects on obese people: insulin resistance and endothelial inflammatory changes, the latter changes the vascular tissue, causing tissue dysfunction and hypertension [20]. Genetic defects, causing leptin deficiency in mice, were associated with increased sensitivity to proinflammatory monocyte/macrophageactivating stimuli, impairment of phagocytic functions, and reduced $\mathrm{T}$-cell function. These mice were reported to have a high susceptibility to bacterial infections (Listeria monocytogenes, Klebsiella pneumonia, etc.) ${ }^{[21-23]}$. In a study comparing the ability of tissues to clear Candida in obese and normal weight rats, it was found that $\mathrm{OB}$ rats had a higher yeast load in their kidneys than non-obese rats within nine days after injection of the intravenous yeast suspension [24]. The validity of these findings in humans has not yet been investigated. In our study, the higher number of hypertensive cases in the UTI group can be explained by the interference between the molecular level of infection cascade and the cascades affecting endothelial damage. However, further studies are needed to distinguish between coincidence and causality.

Okubo et al. ${ }^{[26]}$ showed a higher risk for hypertension in the hospitalized pediatric (<18 years) obese UTI group compared to the non-obese UTI group. It has been shown that obesity may be a risk factor for hypertension and renal parenchymal injury in the absence of VUR. The upper and lower UTIs were not specified in this study. Additionally to this study, our data emphasize that hypertension is associated with UTI in obese participants compared to those without UTI. In our data, although all patients had lower tract infection, hypertension was more frequent in the UTI group then non-UTI group. Renal injury is classically associated with upper UTI in the presence of VUR. Also anatomic, functional (bladder and bowel dysfunction, abnormalities in filling and emptying of the bladder and defects in stool elimination, including constipation and encopresis), or immunologic pathologies (as mentioned above) may cause chronic bacteriuria, recurrent cystitis, or progression to upper urinary tract disease ${ }^{[27-8]}$. In our study, the definitive distinction for upper and lower UTI could not be made due to the lack of information based on the hospital records of patients with recurrent UTIs. Hypertension was shown to be more frequent in the UTI group and this data suggests that these participants have had pyelonephritis previously. Additonaly in an adult 
cliinical study obesity was shown to be a major risk factor for acute renal infarction [29]. Importantly, treatment of UTIs has not been effective at reducing the rate of renal scarring in children ${ }^{[30]}$, therefore studies examining the risk factors that involve renal parenchyma become important. Also there is a need for further studies related to the association of even obesity with parenchymal injury, and the relationship between obesity and infection cascades and hypertension. This study ephasizes the importance of questioning about UTI history and UTI symptomsfor clinical applications of obese patients.

\section{LIMITATIONS}

There are several limitations to our study.Lack of upper and lower discrimination of previous UTI history based on medical records is the limitation of this study. Cohort studies on the subject may be more informative. Also, the absence of nonobese control group is a limitation in terms of our research. However, the prevalence of UTI has been reported in many publications, and we can say that the frequency is higher in obese compared to these studies. Researches are needed on the anatomical differences of obese children and their effects on UTI.The high number of hypertensive cases in the UTI group can be explained by the intervention of cascade affecting endothelial damage at the molecular level of infection cascade. However, further studies are needed to distinguish between coincidence and causality.

\section{CONCLUSION}

The frequency of UTI was $3.39 \%$ in children and adolescents with female predominance who applied for obesity screening. In the obese/overweight UTI group, hypertension and previous history of UTI was shown more common, so it may be important to questionobese/overweight children and adolescents for UTI at their clinical application.

\section{REFFERENCES}

1. Falagas ME, Kompoti M. Obesity and infection. Lancet Infect Dis. 2006; 6:438-46.

2. Wilborn C, Beckham J, Campbell B, Harvey T, Galbreath M, La Bounty P, Nassar E, Wismann J, Kreider R. Obesity: prevalence, theories, medical consequences, management, and research directions. J Int Soc Sports Nutr. 2005; 2:4-31. doi: 10.1186/15502783-2-2-4.

3. Bensman $A$, Dunand $O$, Ulinski $T$. Urinary tract infection, In: Pediatric Nephrology. 2009, 1007-1025. Berlin, Springer.

4. Mahyar A, Ayazi P, Gholmohammadi P, Moshiri SA, Oveisi S, Esmaeily $S$. The role of overweight and obesity in urinary tract infection in children. Infez Med. 2016; 24(1):38-42.

5. Yang TH, Yim HE, Yoo KH. Obesity and a febrile urinary tract infection: dual burden for young children? Urology. 2014; 84(2):445-9. doi: 10.1016/j.urology.2014.03.032.

6. Mahyar A, Ayazi P, Gholmohammadi P, Moshiri SA, Oveisi S, Esmaeily $S$. The role of overweight and obesity in urinary tract infection in children. Infez Med. 2016; 24(1):38-42.

7. Byun HJ, Ha JY, Jung W, Kim BH, Park CH, Kim Cl. The impact of obesity on febrile urinary tract infection and renal scarring in children wit vesicoureteral reflux. J Pediatr Urol. 2017; 13(1):67.e167.e6. doi: 10.1016/j.jpurol.2016.08.018.

8. Grier WR, Kratimenos P, Singh S, Guaghan JP, Koutroulis I.
Obesity as a Risk Factor for Urinary Tract Infection in Children. Clin Pediatr (Phila). 2016; 55(10):952-6. doi: 10.1177/0009922815617974.

9. Okarska-Napierała M, Wasilewska A, Kuchar E. Urinary tract infection in children: Diagnosis, treatment, imaging - Comparison of current guidelines. J Pediatr Urol. 2017; 13(6):567-573. doi: 10.1016/j.jpurol.2017.07.018. Epub 2017 Sep 19. Review.

10. Zeitler P, Arslanian S, Fu J, Pinhas-Hamiel O, Reinehr T, Tandon $\mathrm{N}$, Urakami T, Wong J, Maahs DM. ISPAD Clinical Practice Consensus Guidelines 2018: Type 2 diabetes mellitus in youth. Pediatr Diabetes. 2018; 19 Suppl 27:28-46. doi: 10.1111/pedi.12719.

11. Kuczmarski RJ, Ogden CL, Guo SS, Grummer-Strawn LM, Flegal KM, Mei Z, Wei R, Curtin LR, Roche AF, Johnson CL. 2000 CDC Growth Charts for the United States: methods and development. Vital Health Stat 11. 2002:1-190.

12. National High Blood Pressure Education Program Working Group on High Blood Pressure in Children and Adolescents (2004). The fourth report on the diagnosis, evaluation, and treatment of high blood pressure in children and adolescents. Pediatrics 114:555576.

13. Shaikh N, Morone NE, Bost JE, Farrell MH. Prevalence of urinary tract infection in childhood: a meta-analysis. Pediatr Infect Dis J 2008; 27:302. doi: 10.1097/INF.0b013e31815e4122.

14. Freedman AL. Urologic diseases in North America Project: trends in resource utilization for urinary tract infections in children. J Urol 2005; 173(3):949 - 54.

15. Stein R, Dogan HS, Hoebeke P, Kočvara R, Nijman RJ, Radmayr C, Tekgül S; European Association of Urology; European Society for Pediatric Urology. Urinary tract infections in children: EAU/ESPU guidelines. Eur Urol. 2015; 67:54658.doi:10.1016/j.eururo.2014.11.007.

16. Foxman B. Epidemiology of urinary tract infections: incidence, morbidity, and economic costs. Dis Mon 2003; 49(2):53-70.

17. Faroogi IS, Matarese G, Lord GM, Keogh JM, Lawrence E, Agwu C, Sanna V, Jebb SA, Perna F, Fontana S, Lechler RI, DePaoli AM, O'Rahilly S. Beneficial effects of leptin on obesity, $T$ cell hyporesponsiveness, and neuroendocrine/metabolic dysfunction of human congenital leptin deficiency. J Clin Invest. 2002; 110:1093103.

18. Nieman DC, Henson DA, Nehlsen-Cannarella SL, Ekkens M, Utter $A C$, Butterworth DE, Fagoaga OR. Influence of obesity on immune function. J Am Diet Assoc. 1999; 99:294-9.

19. Yang H, Youm YH, Vandanmagsar B, Rood J, Kumar KG, Butler AA, Dixit VD. Obesity accelerates thymic aging. Blood. 2009 29;114:3803-12. doi: 10.1182/blood-2009-03-213595.

20. Kelishadi R, Roufarshbaf $M$, Soheili S, Payghambarzadeh F, Masjedi M. Association of Childhood Obesity and the Immune System: A Systematic Review of Reviews. Child Obes. 2017; 13(4):332-346. doi:10.1089/chi.2016.0176.

21. Faggioni $R$, Feingold KR, Grunfeld $C$. Leptin regulation of the immune response and the immunodeficiency of malnutrition. FASEB J 2001; 15: 2565-71.DOI:10.1096/fj.01-0431rev.

22. Ikejima S, Sasaki S, Sashinami H, Mori F, Ogawa Y, Nakamura T, Abe Y, Wakabayashi K, Suda T, Nakane A. Impairment of host resistance to Listeria monocytogenes infection in liver of $\mathrm{db} / \mathrm{db}$ and ob/ob mice. Diabetes 2005; 54:182-89.

23. Mancuso P, Gottschalk A, Phare SM, Peters-Golden M, Lukacs NW, Huffnagle GB. Leptin-deficient mice exhibit impaired host defense in Gram-negative pneumonia. J Immunol 2002; 168:401824.

24. Blanc MC, Moinard C, Béziel A, Darquy S, Cynober L, De Bandt JP. Arginine and glutamine availability and macrophage functions in the obese insulin-resistant Zucker rat. J Cell Physiol 2005; 202:153-59.

25. Plotkin BJ, Paulson D, Chelich A, Jurak D, Cole J, Kasimos J, Burdick JR, Casteel N. Immune responsiveness in a rat model for 
type II diabetes (Zucker rat, fa/fa): susceptibility to Candida albicans infection and leucocyte function. J Med Microbiol 1996; 44:277-83.

26. Jain P, Parsons M. A review. The effects of obesity on the pelvic floor. Royal College of Obstetricians and Gynecologists. 2011; 13:133-142.

27. Okubo Y, Handa A. The impact of obesity on pediatric inpatients with urinary tract infections in the United States. J Pediatr Urol. 2017; 13(5):455.e1-455.e5.doi: 10.1016/j.jpurol.2017.03.038. 27.https://0210l1h8s-y-https-www-uptodate.com.cbu.proxy. deepknowledge.io/contents/acute-infectious-cystitis-managementand-prognosis-in-children-older-than-two-years-

andadolescents?search=cystitis\&source=search_result\&selected Title=2 150\& usage_type=defaul \&display_rank=2.

28. Lee T, Park JM. Vesicoureteral reflux and continuous prophylactic antibiotics. Investig Clin Urol. 2017;58(Suppl 1):S32-S37. doi:10.4111/icu.2017.58.S1.S32

29. Mesiano P, Rollino C, Beltrame G, et al. Acute renal infarction: a single center experience. J Nephrol. 2017;30(1):103-107. doi:10.1007/s40620-015-0259-0.

30. Hoberman A, RIVUR Trial Investigators, Greenfield SP, Mattoo TK et al (2014) Antimicrobial prophylaxis for children with vesicoureteral reflux. N Engl J Med 370:2367-2376. https://doi. org/10.1056/NEJMoa1401811. 